\title{
Primary pulmonary artery angiosarcoma mimicking pulmonary embolism in a 66-year-old man with dyspnea
}

\author{
Philippe Desmarais MD, Mikhael Laskine MD MSc, Christine Caporuscio MD
}

$\mathrm{A}$ 66-year-old man consulted his family physician because of progressive dyspnea. He had a history of hypertension and dyslipidemia, but no personal or family history of cardiac or respiratory diseases. He was a lifelong nonsmoker and had been a professional endurance swimmer. After his professional career, he started a swimming school, worked as a trainer and continued to swim on a regular basis. Over the last three years, he noticed a progressive decrease in his endurance. One month before consultation, he experienced a substantial increase in dyspnea and new onset of pleuritic pain localized over the left side of his chest.

The patient was referred to a cardiologist. Results of dipyridamole sestamibi scanning and transthoracic echocardiography were normal. Coronary computed tomography (CT) angiography showed multiple pulmonary emboli, including obstructive lesions in the left pulmonary artery, the left lower lobar artery and the lingular artery (Figure 1). The pulmonary emboli were associated with pulmonary infarctions and a mild left pleural effusion.

The patient was admitted to hospital for investigation. He had no known risk factors for HIV infection and had not used ergogenic drugs. His father had died of an unspecified liver tumour. There was no other family history of cancer. Findings on physical examination were unremarkable. The jugular venous pulse was normal. Heart sounds were normal on cardiac auscultation, with physiologic splitting and a normal pulmonic component of the second heart sound. There was no third heart sound or heart murmur. The liver was not palpable, and there were no signs of deep vein thrombosis or underlying cancer.

Results of blood tests showed mild inflammatory anemia (hemoglobin level 110 [normal 140-180] g/L), a normal leukocyte count and a C-reactive protein level of 60.0 (normal $<5.0$ ) $\mathrm{mg} / \mathrm{L}$. Duplex ultrasonography of the legs was negative for deep vein thrombosis. Repeat transthoracic echocardiography showed normal mor- phology and adequate left and right heart function. The systolic pressure of the pulmonary artery was elevated at $47 \mathrm{~mm} \mathrm{Hg}$.

We diagnosed unprovoked multiple pulmonary emboli with mild pulmonary hypertension. The patient was discharged with a prescription for warfarin, a follow-up visit and referral for an outpatient colonoscopy to investigate the anemia.

The patient's symptoms were not improved one month later. Repeat CT angiography showed an unchanged obstructive lesion in the left pulmonary artery despite the anticoagulation treatment (Figure 2). The lesion measured $5.3 \times 2.6 \mathrm{~cm}$ and was heterogeneously hypodense with intrinsic contrast enhancement. The filling defects in the left lobar and lingular arteries had resolved. The left pleural effusion had markedly increased, and there was now mild dilatation of the right ventricle. These findings suggested a possible malignant process with associated pulmonary emboli. The warfarin was changed to enoxaparin at a therapeutic dose.

Integrated fluorine-18 fludeoxyglucose positron emission tomography/computed tomography (FDG-PET/CT) scan showed that the lesion had high metabolic activity peripherally with a relatively photopenic centre (maximum standardized uptake value of 7.9) (Figure 3). There were no other metabolically active lesions on the scan suggestive of metastatic disease. Bronchoscopy with bronchoalveolar lavage and an endobronchial ultrasonograpy-guided biopsy were also performed. Histopathological examination of the

\section{KEY POINTS}

- Pulmonary embolism can be mimicked clinically and radiologically by a variety of pathological conditions.

- Primary and secondary pulmonary angiosarcomas are rare and highly aggressive tumours associated with a poor prognosis.

- Magnetic resonance imaging and positron emission tomography are useful diagnostic tools to differentiate between pulmonary emboli and pulmonary artery angiosarcomas.

- When surgically feasible, total resection of pulmonary angiosarcomas offers the best long-term prognosis.
Competing interests: None declared.

This article has been peer reviewed.

The authors have obtained patient consent.

Correspondence to:

Philippe Desmarais, philippe desmarais.1@umontreal.ca

CMAJ 2016. DOI:10.1503 /cmaj.151417 
retrieved samples showed strongly atypical cells, suggestive of a neoplastic process.

After three months of anticoagulation treatment, enoxaparin was stopped and the patient underwent extensive curative surgery. The surgery consisted of resection of the left pulmonary artery, reconstruction of the pulmonary trunk and right pulmonary artery with pericardial patch, pulmonary valvuloplasty, radical thymectomy, total pericardiectomy, left pneumonectomy and lymph node dissection. Histopathological examination of the $5.5 \times 3 \times 3 \mathrm{~cm}$ mass confirmed the presence of a high-grade intimal angiosarcoma of the pulmonary trunk (Appendix 1, available at www.cmaj. ca/lookup/suppl/doi:10.1503/cmaj.151417/-/DC1). The immunohistochemistry analysis was positive for several tumour markers, which further supported the diagnosis of pulmonary angiosarcoma. The resected lymph nodes, left lung and pericardium were free of metastatic disease. The patient initially declined chemotherapy and radiotherapy and was discharged home one month later.

A repeat FDG-PET/CT scan five months after discharge showed a new lesion at the left main bronchus, with lymphatic and pulmonary metastasis. These lesions were not amenable to surgery. The patient accepted paclitaxel-based palliative chemotherapy. The chemotherapy drug was later changed to etoposide because of disease progression. Palliative radiotherapy was added to treat a mediastinal bone metastasis. The palliative chemotherapy and radiotherapy were stopped after eight

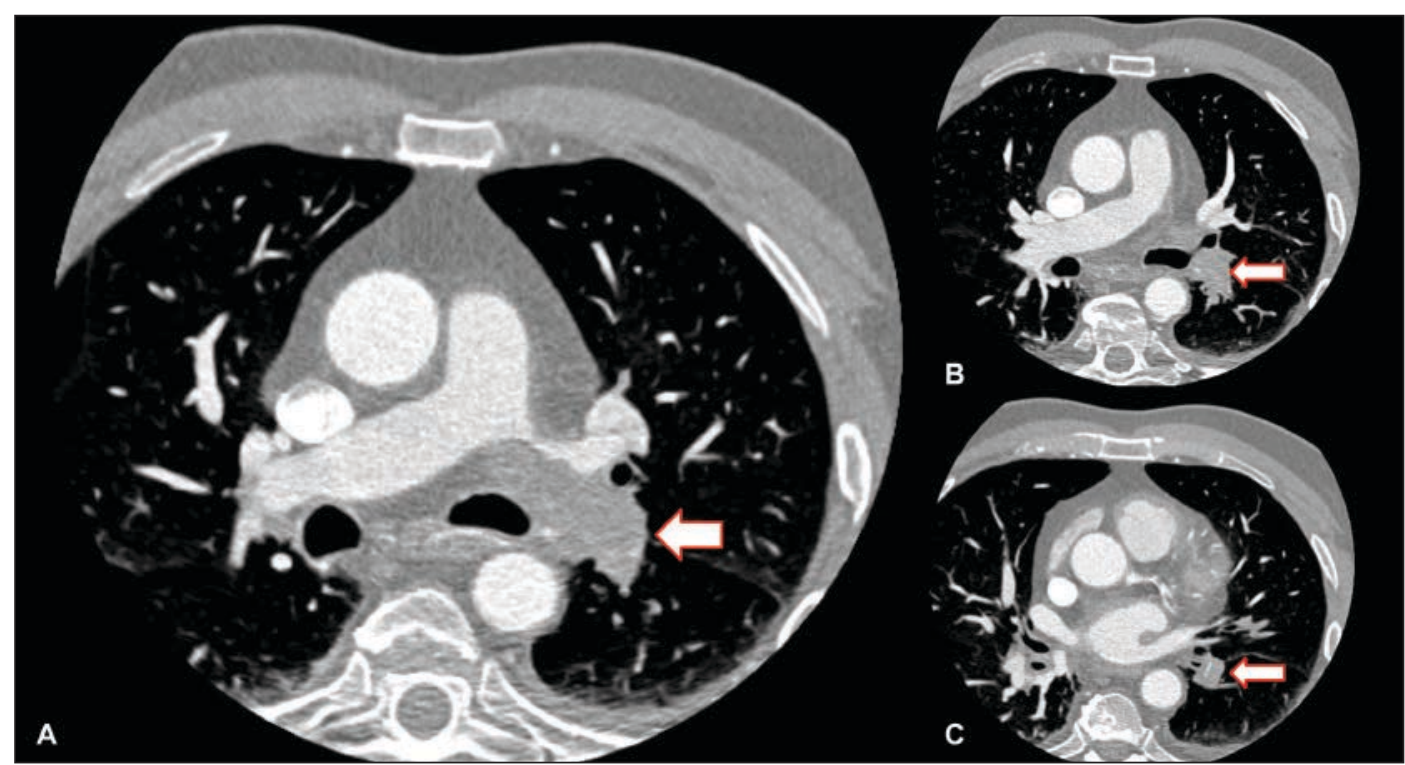

Figure 1: Coronary computed tomography angiograms in a 66-year-old man, showing multiple filling defects (arrows) in the left arterial pulmonary circulation, with a main lesion at the level of the left pulmonary artery.

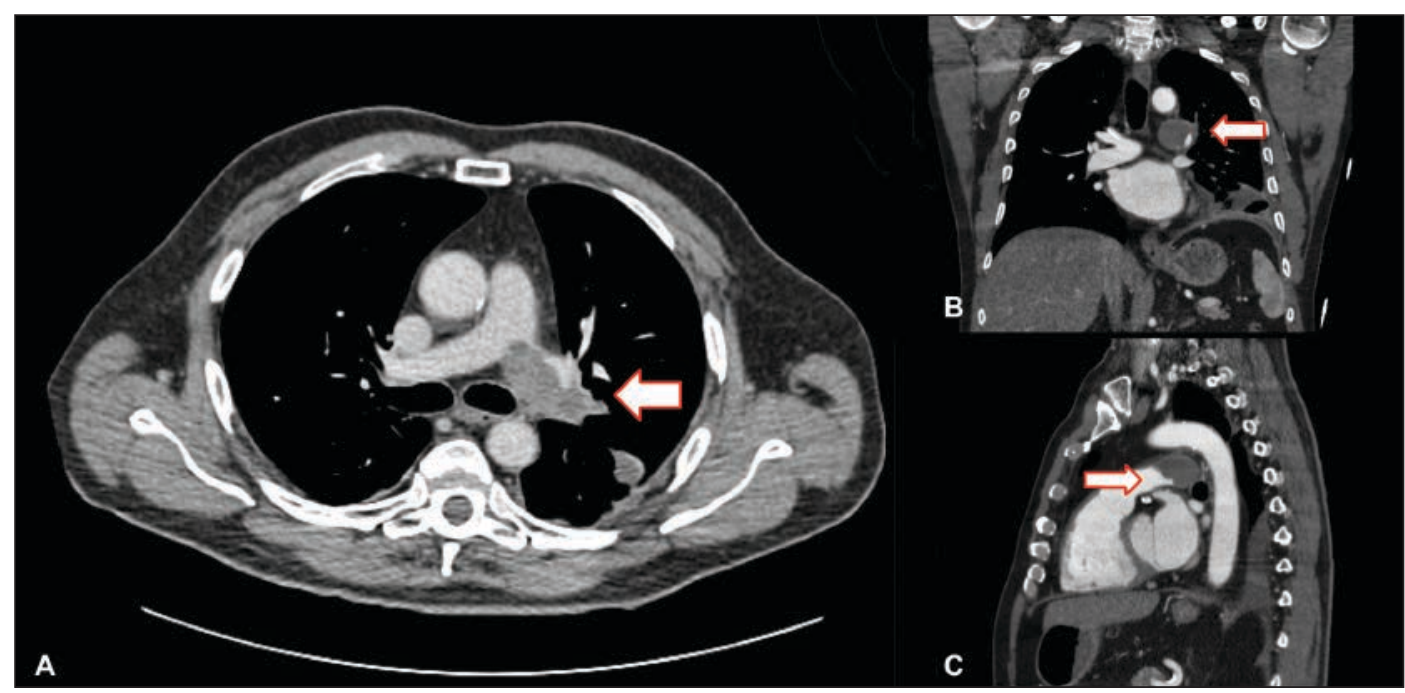

Figure 2: Computed tomography angiograms of the patient's thorax after four weeks of warfarin treatment. A large obstructive lesion (arrows) is visible in the left pulmonary artery, extending into the pulmonary trunk. The lumen of the left pulmonary artery is almost completely obliterated by the lesion. 
months because of poor tumour response and serious adverse effects. The patient was referred to palliative care. He died three months later.

\section{Discussion}

Pulmonary embolism is a frequently encountered condition. Its incidence, although difficult to evaluate, has been estimated to be 112.3 cases per 100000 adults annually since the introduction of CT angiography in $1998 .{ }^{1}$ It is a potentially fatal disease, with an estimated annual mortality of 11.9 deaths per 100000 adults. ${ }^{1}$ About $2.7 \%$ of deaths among all medical admissions are attributed to pulmonary embolism. ${ }^{1}$

Because the presentation of pulmonary embolism is variable and nonspecific, imaging is required for diagnosis. Multiple studies have validated the use of $\mathrm{CT}$ angiography in various clinical settings. It has a sensitivity of $96 \%$ (95\% confidence interval [CI] $82 \%-99 \%$ ) and a specificity of $86 \%$ (95\% CI 67\%-96\%) for the diagnosis of acute pulmonary embolism in outpatient settings. ${ }^{2}$ The use of CT angiography is preferred to a ventilationperfusion scan and conventional pulmonary angiography for technical, availability and patient-related reasons. Although highly accurate, CT angiography may show a variety of conditions that mimic pulmonary emboli (Box 1). Primary and secondary invasive tumours of the pulmonary arteries, such as angiosarcomas, are uncommon pathological causes of pseudoemboli seen on imaging.

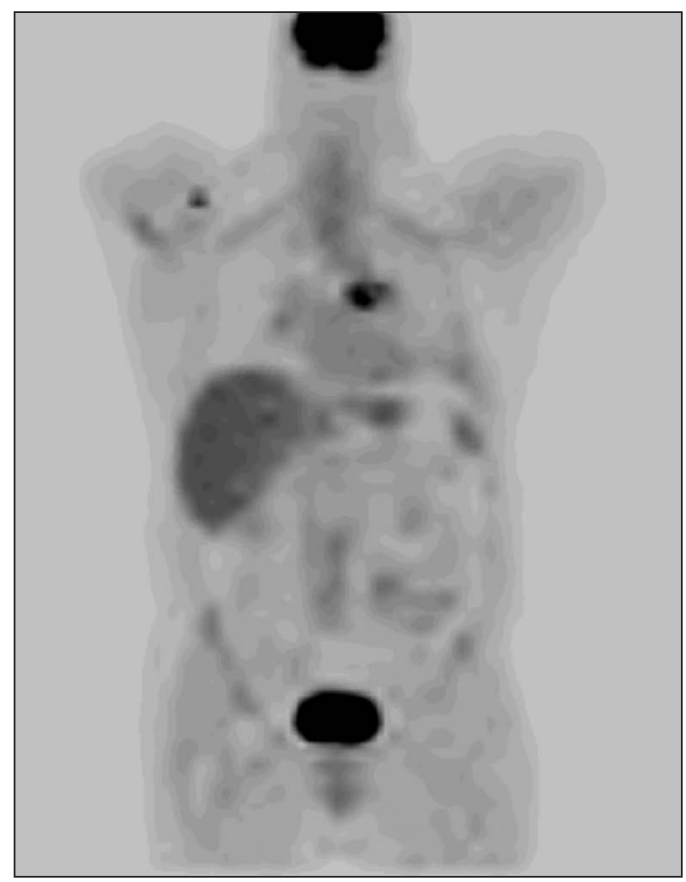

Figure 3: Integrated fluorine-18 fludeoxyglucose positron emission tomography/computed tomography scan showing lesion with high metabolic activity at the level of the pulmonary artery.

\section{Pulmonary artery angiosarcomas}

Sarcomas are rare tumours of mesenchymal origin. They originate in bones or in soft tissues such as cartilage, fat, muscle and endothelial cells. Angiosarcomas arise from endothelial cells and comprise only $2 \%$ of soft-tissue sarcomas. ${ }^{3}$ They are classified as cutaneous angiosarcoma, lymphedema-associated angiosarcoma, radiation-induced angiosarcoma, primary breast angiosarcoma and soft-tissue angiosarcoma. Cutaneous angiosarcomas are the most common subtype, often occurring on the head and neck. ${ }^{3}$

Angiosarcomas can occur in any endothelial tissue of the body, including the heart, lungs, aorta and the great vessels. In these anatomic locations, they can cause serious obstructive complications such as cardiac tamponade, limb claudication and pulmonary hypertension. ${ }^{4}$

There have been fewer than 250 cases of primary pulmonary sarcoma described in the literature. These include angiosarcomas and other subtypes of pulmonary artery sarcomas, such as chondrosarcomas, liposarcomas and rhabdomyosarcomas. ${ }^{5}$ It is estimated that $3.6 \%$ of pulmonary sarcomas are angiosarcomas. ${ }^{6}$ Pulmonary artery sarcomas usually originate from the intimal cells and are referred to as intimal sarcomas. Histologically, intimal sarcomas consist of poorly differenti-

Box 1: Causes of pseudoembolism on computed tomography (CT) angiography $y^{2,3,8}$

Anatomic

- Calcified lymph nodes

- Enlarged hilar lymph nodes

- Venous collateral circulation

- Venous filling defect

Pathological

- Aspiration

- Endobronchial tumour

- Thrombus in left atrial appendage

- Thrombus in pulmonary vein

- Invasion of pulmonary arteries by primary or secondary tumour

- Mucous bronchogram

latrogenic

- Catheter

- Foreign body

- Postsurgical change

Technical

- CT scanner artifact

- Edge enhancement

- Quantum mottle

- Streak artifact

- Transient interruption of contrast

- Window setting 
ated mesenchymal cells, with fibroblastic and myofibroblastic components. Pulmonary artery angiosarcomas typically arise from the pulmonary trunk. ${ }^{5}$ They metastasize regionally to the lung and mediastinal lymph nodes, and distally through hematogenous dissemination.

\section{Predisposing factors}

Although the exact pathophysiology of angiosarcomas is unknown, there are well-established predisposing factors for their development. The association between radiotherapy and angiosarcomas has been described in patients with breast cancer. An epidemiologic study involving 194798 patients showed a peak incidence of soft-tissue sarcomas a decade after adjuvant radiotherapy for breast cancer. ${ }^{7}$ This association has been described in patients receiving treatment for other cancers as well. Stewart-Treves syndrome refers to the development of angiosarcomas in patients with chronic lymphedema. Chronic lymphedema secondary to surgery, radiotherapy or infection such as filariasis have all been linked to the occurrence of angiosarcomas. ${ }^{3}$

Exposure to exogenous toxins can predispose patients to angiosarcomas, typically in the liver. ${ }^{3}$ Culprit toxins include arsenic, anabolic steroids, vinyl chloride and thorium dioxide. ${ }^{3}$

Neurofibromatosis type 1, Maffucci syndrome, Klippel-Trénaunay syndrome and some genetic mutations (e.g., BRCA1 and BRCA2) are also associated with angiosarcoma development. ${ }^{3}$

Lastly, immunosuppression is another suspected predisposing factor. A handful of cases have been described in chronically immunosuppressed recipients of renal transplants and in patients with AIDS. ${ }^{3}$

\section{Clinical presentation and diagnosis}

The clinical presentation of pulmonary artery angiosarcomas is nonspecific. Most common symptoms are dyspnea, pleuritic chest pain, cough and hemoptysis. ${ }^{6} 8$ Patients may have signs of chronic pulmonary hypertension. Because the radiologic findings resemble those of subacute pulmonary embolism, angiosarcomas are often misdiagnosed as pulmonary embolism. 6,8

On CT angiography, findings of tumours in the pulmonary vasculature include low attenuation filling defects, heterogeneous enhancement of the mass in the lumen of the artery and extravascular spread of the lesion. ${ }^{9}$ Gadoliniumenhanced magnetic resonance imaging may help differentiate between thrombotic masses and vasculature tumours. Unlike thromboemboli, angiosarcomas exhibit heterogeneous enhancement with gadolinium. ${ }^{5}$ The use of FDG-PET/CT helps differentiate between malignant and benign pulmonary artery lesions. A study involving 18 patients with pulmonary artery filling defects on CT angiography used a standardized uptake value cutoff for PET of 3.5. This cutoff had a sensitivity, specificity and accuracy of $100 \%$ for the detection of malignant lesions. ${ }^{9}$

\section{Treatment and prognosis}

Radical surgical resection offers the best prognosis, with a median survival of $36.5 \pm 20.2$ months. ${ }^{10}$ Subtotal resection or debulking of the tumour offers a median survival of $11 \pm 3$ months. ${ }^{10}$ Unfortunately, many patients are not surgical candidates because as many as $50 \%$ present with metastatic disease. ${ }^{4}$ Without surgery, the mean survival is 1.5 months. ${ }^{8}$ Chemotherapy may prolong the median survival time, but supporting evidence is limited. ${ }^{4,8,10}$

\section{Conclusion}

The diagnosis of pulmonary angiosarcomas is challenging. These tumours are rare and mimic pulmonary emboli, which are much more common. Our patient's condition did not improve with therapeutic anticoagulation, and atypical findings appeared on follow-up imaging. In such cases, it is appropriate to consider causes of pseudoembolism on CT imaging.

\section{References}

1. Wiener RS, Schwartz LM, Woloshin S. Time trends in pulmonary embolism in the United States: evidence of overdiagnosis. Arch Intern Med 2011;171:831-7.

2. Coche E, Verschuren F, Keyeux A, et al. Diagnosis of acute pulmonary embolism in outpatients: comparison of thin-collimation multi-detector row spiral CT and planar ventilation-perfusion scintigraphy. Radiology 2003;229:757-65.

3. Young RJ, Brown NJ, Reed MW, et al. Angiosarcoma. Lancet Oncol 2010;11:983-91.

4. Fatima J, Duncan AA, Maleszewski JJ, et al. Primary angiosarcoma of the aorta, great vessels, and the heart. J Vasc Surg 2013; 57:756-64.

5. Huo L, Lai S, Gladish G, et al. Pulmonary artery angiosarcoma: a clinicopathologic and radiological correlation. Ann Diagn Pathol 2005;9:209-14.

6. Nakamura Y, Shimizu T, Fukumoto Y, et al. A case of angiosarcoma arising in the trunk of the right pulmonary artery clinically simulating pulmonary embolism. World J Oncol 2012;3:119-23.

7. Huang J, Mackillop WJ. Increased risk of soft tissue sarcoma after radiotherapy in women with breast carcinoma. Cancer 2001;92: 172-80.

8. Kim JB, Kim SH, Lim SY, et al. Primary angiosarcoma of the pulmonary trunk mimicking pulmonary thromboembolism. Echocardiography 2010;27:E23-6.

9. Lee EJ, Moon SH, Choi JY, et al. Usefulness of fluorodeoxyglucose positron emission tomography in malignancy of pulmonary artery mimicking pulmonary embolism. ANZ J Surg 2013;83:342-7.

10. Blackmon SH, Rice DC, Correa AM, et al. Management of primary pulmonary artery sarcomas. Ann Thorac Surg 2009;87:977-84.

Affiliations: Geriatric Service (Desmarais) and Internal Medicine Service (Laskine, Caporuscio), Department of Medicine, Centre Hospitalier de l'Université de Montréal, Montréal, Que.

Contributors: Christine Caporuscio and Philippe Desmarais drafted the manuscript. All of the authors contributed to the literature review, revised the manuscript critically for important intellectual content, approved the final version to be published and agreed to act as guarantors of the work. 